Ann. Biol. anim. Bioch. Biophys., I972, 12 (2), 289-306.

\title{
EFFETS ISOLÉS ET COMBINÉS DES ACIDES ACÉTIQUE, PROPIONIQUE, LAURIQUE ET STÉARIQUE SUR LA SÉCRÉTION LIPIDIQUE DE LA MAMELLE DE GHÈVRE
}

\author{
P. M. FEHR, D. SAUVANT et J. DELAGE \\ avec la collaboration technique de J. Hervieu, Annie Rouzeau, \\ Michèle DorLEAns et Nicole Sargousse \\ Laboratoire de Recherches de la Chaire de Zootechnie, I. N. R. A., \\ Institut National Agronomique \\ 16, rue Claude-Bernard, Paris $5^{\mathrm{e}}$
}

\section{RÉSUMÉ}

Au cours de deux expériences $\mathrm{F}$ et $\mathrm{C}$ réalisées en carré latin sur $\mathbf{I} 8$ chèvres, les effets isolés et certains effets combinés des acides acétique, propionique, laurique et stéarique sur la production et la composition du lait, sur la composition en acides gras de la matière grasse du lait et sur leurs quantités sécrétées par jour ont été étudiés.

Dans chaque expérience, six régimes sont distribués aux chèvres. Le régime témoin $T$ de l'expérience $F$ est pauvre en lipides et bien pourvu en fourrages; celui de l'expérience $C$ est pauvre en fourrages et un peu moins carencé en lipides. On ajoute au régime $T$ de l'acétate de sodium, du stéarate d'éthyl, de l'acétate et du stéarate simultanément dans les deux expériences, du propionate de sodium, du propionate et du stéarate ensemble dans l'expérience $F$, du laurate d'éthyl, de l'acétate et du laurate ensemble dans l'expérience C.

L'addition de stéarate au régime $T$ augmente le taux butyreux, la sécrétion de matières grasses du lait, les pourcentages et les quantités sécrétées des acides stéarique et oléique. Les teneurs des acides à plus courte chaîne ont tendance à baisser. Le stéarate alimentaire semble ralentir la synthèse intra-mammaire des acides gras. Ces effets sont moins marqués dans l'expérience $C$ que dans l'expérience $F$.

Le laurate tend à diminuer les productions de lait, de matières grasses et de matières azotées ainsi que les quantités sécrétées d'acides gras, excepté celle d'acide laurique qui augmente nettement. La mamelle sécréterait d'autant plus facilement un acide gras d'origine alimentaire que sa chaîne est longue. Les effets du laurate et de l'acétate semblent indépendants.

Le propionate a peu d'influence. Il diminue légèrement la quantité de matières grasses sécrétées en raison d'une sécrétion plus faible d'acides gras à moins de 18 atomes de carbone. Par ailleurs, il augmente le taux de matières azotées.

Comme le stéarate, l'acétate accroît le taux butyreux et la production de matières grasses, mais de façon plus marquée dans l'expérience $\mathrm{C}$ que dans l'expérience $\mathrm{F}$. Il semble bien que la mamelle utilise d'autant mieux un précurseur de ses matières grasses qu'elle en est carencée. A la suite de l'addition d'acétate, les quantités sécrétées de tous les acides gras du lait, en particulier les acides en $\mathrm{C}_{\mathbf{1 8}}$, augmentent. L'interaction des effets acétate et stéarate semble dépendre de la nature du régime témoin. Lorsque le régime carence nettement la mamelle à la fois en acide acétique et en acides gras longs, les effets de l'acétate et de stéarate s'ajoutent. Si la mamelle est peu déficitaire en acide acétique, leurs effets cumulés s'atténuent et ne sont plus additifs. Comme les acides gras à chaîne longue limiteraient la synthèse intra-mammaire à partir d'acide acétique, ces acides gras longs alimentaires auraient un effet plus prononcé que l'acide acétique sur la sécrétion lipidique de la mamelle de chèvre. 


\section{INTRODUCTION}

L'origine des acides gras du lait de ruminant est actuellement bien établie

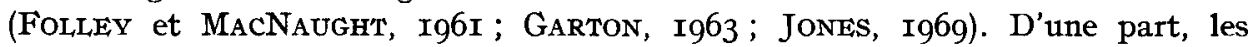
acides gras à $I 8$ atomes de carbone dont la majeure partie est d'origine alimentaire (DELAGE et FEHR, I967c) sont directement prélevés par la mamelle à partir des triglycérides des chylomicrons et des $\beta$-lipoprotéines du plasma sanguin. D'autre part, les acides gras à plus courte chaîne contenant de 4 à $\mathrm{I}_{4}$ atomes de carbone sont synthétisés de novo dans la mamelle à partir de l'acide acétique et, à un moindre degré, de 1'acide $\beta$-hydroxybutyrique. L'acide acétique et l'acide butyrique transformé dans la paroi du rumen et dans le foie en acide $\beta$-hydroxybutyrique, se forment au cours des fermentations du rumen. La production de l'acide acétique est favorisée par les aliments cellulosiques du régime et surtout par les fourrages non broyés. L'acide palmitique du lait peut provenir des lipides alimentaires et aussi être synthétisé dans là mamelle.

L'importance relative des acides gras d'origine alimentaire et des acides gras synthétisés par la mamelle dans la formation des lipides du lait dépend de nombreux facteurs qui sont mal connus (GARTON, I963). Dans ces conditions, nous avons recherché l'influence sur l'utilisation par la mamelle de l'acide acétique et des acides en $\mathrm{C}_{18}$ :

- de l'addition séparée et simultanée au régime d'acide acétique et d'un acide alimentaire qui ne subit pas de transformations dans le rumen, l'acide stéarique, ce qui permet d'apprécier l'effet de chacun de ces deux précurseurs sur l'utilisation de l'autre pour la synthèse des lipides du lait ;

- de l'addition d'acide propionique considéré généralement comme défavorable à la sécrétion lipidique (ARMSTRONG, I968) ;

- de l'addition d'acide laurique qui peut être aussi synthétisé par la mamelle ;

- de la nature du régime de base, selon qu'il apporte à la mamelle des 'quantités faibles ou élevées d'acide acétique.

Dans ce but, au cours de deux expériences, nous avons distribué à des chèvres en lactation des régimes pauvres en lipides et à teneur différente en cellulose brute et nous y avons ajouté ou non divers acides gras seuls ou associés.

Une partie de cette étude a déjà été rapportée par ailleurs (FEHR et DELAGE, I970).

\section{MATÉRIEL ET MÉTHODES}

Dans deux expériences $\mathrm{F}$ et $\mathrm{C}$ réalisées suivant le dispositif de carré latin, dix-huit chèvres adultes de race Alpine, au troisième mois de lactation, sont réparties en six lots comparables. Elles reçoivent successivement au cours de six périodes de trois semaines l'un des six régimes expérimentaux suivants :

\begin{tabular}{ll}
\multicolumn{1}{c}{ Expérience F } & \multicolumn{1}{c}{ Expérience C } \\
Régime Témoin T & Régime Témoin \\
Régime Acétate A & Régime Acétate A \\
Régime Stéarate S & Régime Stéarate S \\
Régime Propioniate P & Régime Laurate L \\
Régime Acétate-Stéarate AS & Régime Acétate-Stéarate AS \\
Régime Propionate-Stéarate PS & Régime Acétate-Laurate AL
\end{tabular}


Les compositions des rations et des aliments concentrés sont rapportées respectivement dans les tableaux I et 2 . Quel que soit le régime, les rations de l'expérience $C$ sont composées de $20 \mathrm{p}$. Ioo de fourrages non broyés et de $80 \mathrm{p}$. roo d'aliments concentrés, alors que celles de l'expérience $\mathrm{F}$ contiennent $40 \mathrm{à} 53 \mathrm{p}$. Ioo de fourrages non broyés et 47 à $60 \mathrm{p}$. roo d'aliments concentrés. Les chèvres reçoivent ainsi des rations dont la matière sèche totale renferme 25 à $30 \mathrm{p}$. IoO de cellulose brute dans l'expérience $\mathrm{F}$ et seulement $12 \mathrm{p}$. Ioo dans l'expérience $\mathrm{C}$. Par ailleurs, en raison de la faible teneur en extrait éthéré des régimes témoins (o,8-I,I p. Ioo dans l'expérience $F$ et de I,2-I,5 p. Ioo dans l'expérience $C$ ) et compte tenu de la consommation de matière sèche, les chèvres n'ingèrent respectivement que 24,2 et $26,2 \mathrm{~g}$ de matières grasses par jour. Ainsi, le régime témoin de l'expérience $\mathrm{F}$ peut être considéré comme pauvre en lipides et assez bien pourvu en cellulose brute, tandis que celui de l'expérience $C$ est pauvre en cellulose brute et légerement moins carencé en matières grasses que le régime correspondant de l'expérience $F$.

\section{TABLEAU I}

Composition des rations

(en kg par jour et par chèvre)

\begin{tabular}{|c|c|}
\hline \multicolumn{2}{|l|}{ (Expérience F) } \\
\hline \multicolumn{2}{|l|}{ Ration de base : } \\
\hline $\begin{array}{l}\text { - Paille de blé, } \ldots \ldots \ldots \ldots \ldots \ldots \ldots \ldots \ldots \\
\text { - Foin de luzerne } \ldots \ldots \ldots \ldots \ldots \ldots \ldots \ldots \\
\text { - Aliment concentre expérimental } \ldots \ldots \ldots\end{array}$ & $\begin{array}{l}0,7 \mathrm{~kg} \\
0,5 \mathrm{~kg} \\
1,1 \mathrm{~kg}\end{array}$ \\
\hline \multicolumn{2}{|l|}{ Au-dessus d'une production de $2 \mathrm{~kg}$ de lait : } \\
\hline \multicolumn{2}{|l|}{ (Expérience C) } \\
\hline \multicolumn{2}{|l|}{ Ration de base : } \\
\hline $\begin{array}{l}\text { - Foin de luzerne } \ldots \ldots \ldots \ldots \ldots \ldots \ldots \\
\text { - Pulpes de betteraves déshydratées } \ldots \ldots \\
\text { - Aliment concentré expérimental } \ldots \ldots\end{array}$ & $\begin{array}{l}0,3 \mathrm{~kg} \\
0,4 \mathrm{~kg} \\
1,1 \mathrm{~kg}\end{array}$ \\
\hline \multicolumn{2}{|l|}{ Au-dessus d'une production de $2 \mathrm{~kg}$ de lait : } \\
\hline $\begin{array}{l}\text { - Foin de luzerne } \ldots \ldots \ldots \ldots \ldots \ldots \ldots \\
\text { - Aliment concentré } " \text { lactation } » \ldots \ldots \ldots\end{array}$ & $\begin{array}{l}0,05 \mathrm{~kg} / \mathrm{kg} \text { de lait } \\
0,3 \mathrm{~kg} / \mathrm{kg} \text { de lait }\end{array}$ \\
\hline
\end{tabular}

Les acides acétique, propionique, laurique et stéarique sont introduits dans le régime sous la forme d'acétate et de propionate de sodium, de laurate et de stéarate d'éthyl. Ils se substituent à l'amidon de maïs et à la paille ou au son de blé dans l'aliment concentré expérimental suivant une équivalence en énergie métabolisable de telle façon que le niveau énergétique et la teneur en matières azotées de tous les aliments concentrés expérimentaux soient identiques dans chaque expérience. Les animaux reçoivent quotidiennement $58 \mathrm{~g}$ d'acétate ou de propionate et $52 \mathrm{~g}$ de stéarate dans l'expérience $\mathrm{F}$ et, $62 \mathrm{~g}$ d'acétate et $47 \mathrm{~g}$ de stéarate ou de laurate dans l'expérience $\mathrm{C}$.

Les rations des animaux sont établies suivant les recommandations de FEHR et DisseT (1969). Les chèvres placées sur caillebotis sont alimentées individuellement par deux repas quotidiens de fourrages et d'aliments concentrés et sont traites deux fois par jour. Elles sont habituées au régime témoin environ un mois avant le début de chaque expérience. Les changements de régime s'étalent sur quatre repas.

Les quantités d'aliments réellement ingérées et la production laitière sont enregistrées tous les jours. Le taux butyreux (méthode Gerber) et le taux de matières azotées (méthode du noir 
amido) sont mesurés sur un échantillon moyen représentatif de quatre traites sept fois par période.

Trois prélèvements moyens de lait, effectués entre le $\mathrm{I}_{\mathrm{I}}{ }^{\mathrm{e}}$ et le $2 \mathrm{I}^{\mathrm{e}}$ jour de chaque période sont utilisés pour la détermination de la composition en acides gras de $4: 0$ à $18: 3$ des matières grasses du lait par chromatographie en phase gazeuse. La description générale des méthodes utilisées a déjà été rapportée (DELAGE et FEHR, I967 a). Les matières grasses du lait sont extraites par la méthode Rose-GotTlieb (1926) et méthylées suivant la méthode de Craig et MurTy (I959). Les esters méthyliques des acides gras sont chromatographiés sur un appareil Girdel S 75 muni d'un détecteur à ionisation de flamme. La colonne est constituée de chromosorb DMCS (80Ioo mesh) imprégné de $4 \mathrm{p}$. Ioo de succinate de di-éthylène glycol.

\section{TABLEAU 2}

Composition des aliments concentrés

\begin{tabular}{|c|c|c|c|c|}
\hline & \multicolumn{2}{|c|}{ Expérience F } & \multicolumn{2}{|c|}{ Expérience C } \\
\hline & $\begin{array}{l}\text { Concentré } \\
\text { expérimental } \\
\text { du régime } \\
\text { Témoin }\end{array}$ & $\begin{array}{l}\text { Concentré } \\
\text { "lactation" }\end{array}$ & $\begin{array}{l}\text { Concentré } \\
\text { expérimental } \\
\text { du régime } \\
\text { Témoin }\end{array}$ & $\begin{array}{l}\text { Concentré } \\
\text { " lactation " }\end{array}$ \\
\hline \multicolumn{5}{|l|}{ En pourcentage } \\
\hline Paille de blé $\ldots \ldots \ldots \ldots \ldots \ldots$ & 29 & 5 & 0 & 0 \\
\hline Blé $\ldots \ldots \ldots \ldots \ldots \ldots \ldots$ & 10 & 5 & 0 & 0 \\
\hline Orge $\ldots \ldots \ldots, \ldots, \ldots, \ldots, \ldots$ & 0 & 0 & 30 & 70 \\
\hline Amidon de mais...$\ldots$. & 38 & 50 & 30 & 0 \\
\hline Son de ble............. & 0 & 0 & 22 & 0 \\
\hline Tourteau de soja $\ldots \ldots \ldots \ldots$ & 25 & 35 & 13 & 25 \\
\hline Composé minéral vitaminique . & 5 & 5 & & 5 \\
\hline $\mathrm{UF}, \ldots \ldots \ldots \ldots \ldots \ldots \ldots$ & 0,78 & 0,96 & 0,93 & 0,96 \\
\hline M.A.D en $g \quad \ldots \ldots \ldots \ldots \ldots$ & 115 & 160 & 119 & 157 \\
\hline
\end{tabular}

En raison de l'erreur effectuée dans l'interprétation du chromatogramme en deçà de l'acide 8 : o et au-delà de l'acide $\mathrm{I} 8: 2$, nous avons jugé préférable de ne pas présenter les résultats des acides $4: 0,6:$ o et $18: 3$ mais d'en tenir compte dans la somme totale des acides gras sécrétés. Chaque acide est ainsi exprimé en pourcentage de son ester éthylique rapporté à la somme des esters méthyliques des acides de $4:$ o à $18: 3$.

Les résultats du carré latin ont été interprétés par analyse de variance. Nous avons rangé les moyennes correspondant à chaque traitement suivant le test dit de "classement des moyennes ". Comme le dispositif expérimental utilisé le permet, les interactions entre les effets acétate et stéarate, les effets propionate et stéarate et les effets acétate et laurate ont été recherchées. L'ensemble de l'interprétation statistique a été réalisée suivant les méthodes de CochraN et Cox (I957).

\section{RÉSULTATS}

D’après l'analyse de variance du carré latin sur I08 données ( 18 chèvres $\times 6$ périodes), l'effet dû au régime comme les effets dus à la période et aux animaux sur la production et la composition du lait, le pourcentage et la quantité sécrétée journalière d'acides gras se sont révélés significatifs au cours des expériences $\mathrm{F}$ et $\mathrm{C}$, sauf dans les rares cas que nous mentionnons ci-après. 


\section{Expérience $F$}

Consommation alimentaire (tab1. 3).

La consommation totale de matière sèche des chèvres n'a guère varié en fonction de la nature du régime. Les aliments concentrés expérimentaux malgré leur " flaveur " due aux acides qu'ils contiennent sont très bien acceptés. L'aliment renfermant le propionate seul, est le moins bien consommé, mais de façon non significative.

Production et composition du lait (fig. I).

La nature du régime n'influence pas significativement la production laitière des chèvres. Cependant, comme l'indique la figure $I$, les régimes qui ont tendance à la favoriser contiennent du stéarate. Par rapport au régime Témoin, le régime Acétate tend légèrement à augmenter la production du lait et le régime Propionate a une tendance opposée.

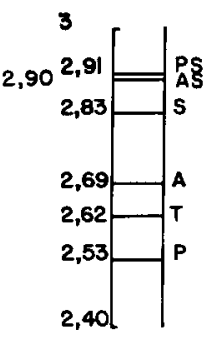

Production laitiere $(\mathrm{kg})$

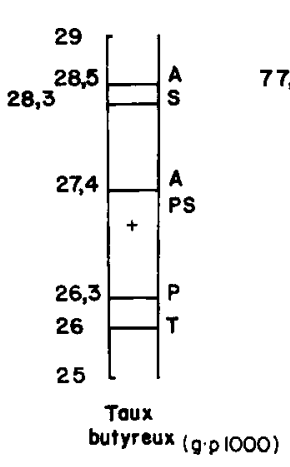

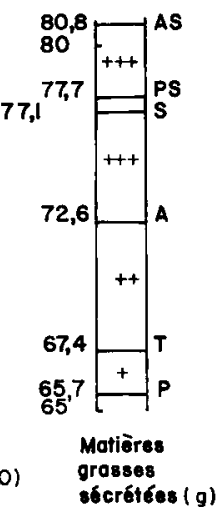

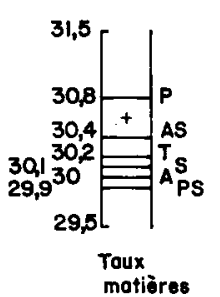

azotóes (g.p 1000)

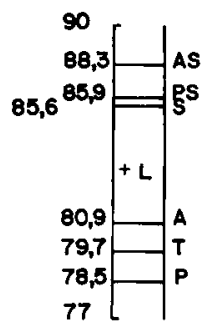

Motiòres ozol 6es scrétóes $(\mathrm{g})$

FIG. I. - Influence du régime sur la production et la composition du lait

(Expérience $\mathrm{F}$ )

$$
\text { Régimes }\left\{\begin{array}{l}
\text { T : Témoin } \\
\text { A : Acétate } \\
\text { S : Stéarate } \\
\text { P : Propionate } \\
\text { AS : Acétate-Stéarate } \\
\text { PS : Propionate-Stéarate }
\end{array}\right.
$$

Les différents régimes sont classés pour chaque variable selon l'ordre décroissant des résultats. Lorsque les deux régimes sont séparés :

$$
\operatorname{par}\left\{\begin{array}{ccc} 
\pm & \text { la différence de leurs résultats est significative à } P<0,1 \\
+ & \text { la différence de leurs résultats est significative à } P<0, \text { o5 } \\
++ & \text { la différence de leurs résultats est significative à } P<0,0 \text { I } \\
+++ & \text { la différence de leurs résultats est significative à } P<0,00 \text { I }
\end{array}\right.
$$

L : signifie à la limite de signification.

L'addition d'acétate et surtout de stéarate au régime s'accompagne d'une augmentation du taux butyreux et d'un accroissement plus net de la quantité de matières grasses sécrétées, alors que le propionate a un effet négligeable, et même plutôt défavorable sur le taux butyreux quand il est ajouté au régime Stéarate.

Les variations du taux de matières azotées sont peu importantes. Toutefois, avec le régime Propionate il est significativement plus élevé. La nature des régimes 
paraît influencer de la même manière les quantités de matières azotées et de matières grasses sécrétées quoique les variations sur les matières azotées soient moins prononcées.

Pourcentage et quantités d'acides gras sécrétés (fig. 2 et 3 ).

L'effet du régime sur le pourcentage des acides gras et sur leurs sécrétions journalières est toujours significatif excepté sur les quantités sécrétées d'acides laurique et linoléique. Les fluctuations les plus importantes sont enregistrées avec les régimes contenant du stéarate. En effet, dans ce cas, comme le montrent les figures 2 et 3 , le pourcentage et la sécrétion journalière d'acide palmitique baissent alors que les teneurs et les quantités sécrétées des acides stéarique et oléique

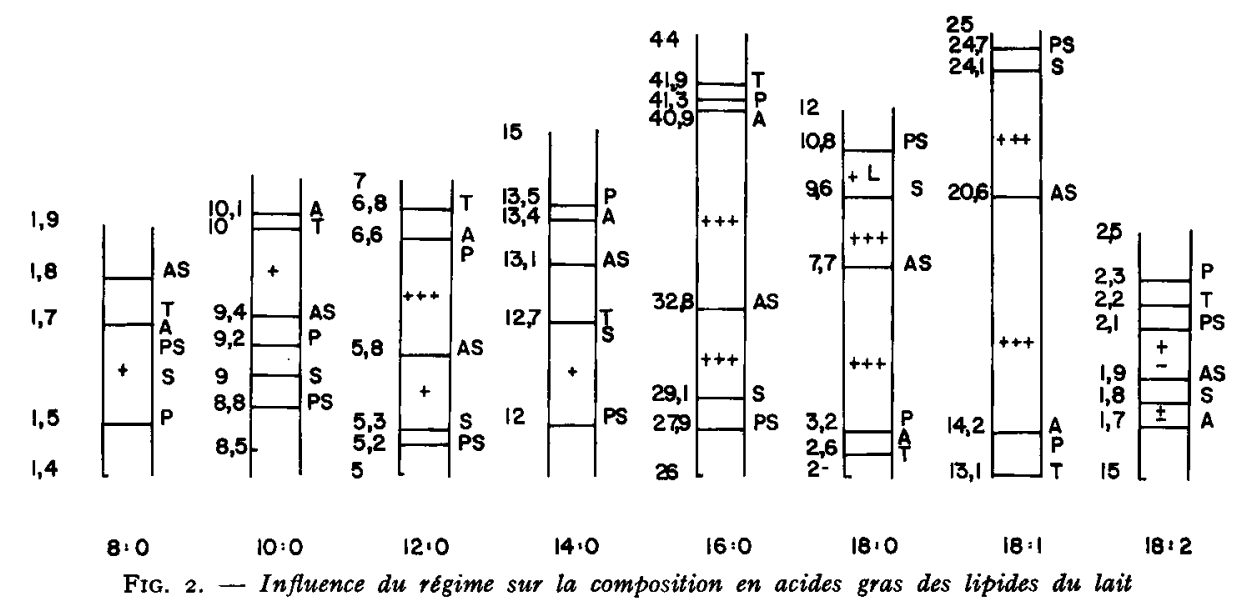

(Expérience $\mathrm{F}$ )

(Légende, cf. fig. I)
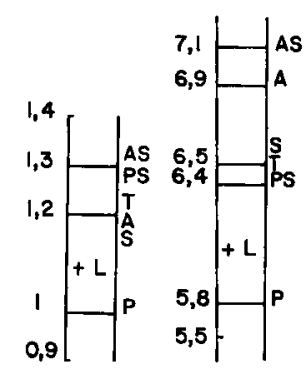

8:0

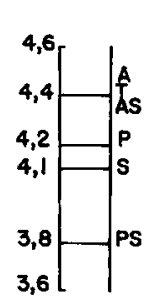

$12: 0$

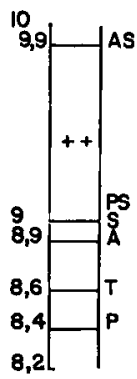

14:0

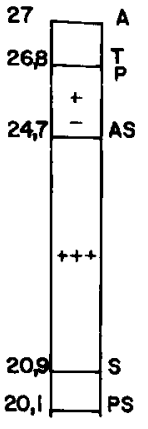

$16: 0$

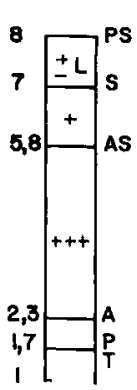

18.0

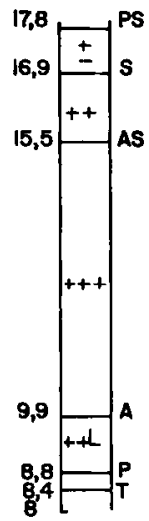

18.1

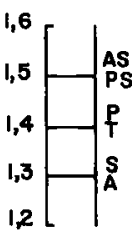

$18: 2$

Fig. 3. - Infuence du régime sur les quantités sécrétées d'acides gras dans le lait

(Expérience F)

(Légende, cf. fig. I) 
augmentent significativement. En présence de stéarate dans le régime, les pourcentages des acides gras à moins de I4 atomes de carbone ont tendance à être inférieurs à ceux du régime Témoin. En revanche, leurs quantités produites par jour sont égales ou même légèrement supérieures avec le régime AcétateStéarate à celles qui sont sécrétées avec le régime Témoin.

L'acétate et le propionate ajoutés seuls modifient peu la composition en acides gras des matières grasses du lait. Cependant, ces deux acides augmentent de façon non significative les pourcentages des acides stéarique et oléique. Par ailleurs, en présence de stéarate, l'acétate tend à limiter l'effet du stéarate et le propionate à le rendre encore plus net.

L'acétate provoque l'accroissement de la sécrétion journalière de tous les acides gras sauf des acides caprylique et laurique. La quantité supérieure de matières grasses sécrétées avec le régime Acétate est en partie due à une sécrétion plus intense des acides stéarique et oléique. Par ailleurs, la légère diminution de la sécrétion lipidique à la suite de l'introduction de propionate seul s'explique plutôt par une quantité sécrétée un peu plus faible d'acides à moins de 16 atomes de carbone.

Les effets de l'acétate et du stéarate dans le régime Acétate-Stéarate semblent s'ajouter sauf dans le cas des pourcentages et des productions des acides palmitique stéarique et oléique pour lesquels leur interaction est significative (tabl. 4). En effet en présence de stéarate, l'acétate du régime abaisse les pourcentages des acides stéarique et oléique et élève celui de l'acide palmitique, tandis qu'il a plutôt un effet opposé quand il est ajouté seul au régime Témoin.

\section{Expérience $C$}

Consommation alimentaire (tabl. 3).

L'appétit des chèvres est plus variable dans l'expérience $C$ que dans l'expérience $\mathbf{F}$; ce qui peut s'expliquer par la faible proportion d'aliments grossiers non broyés distribués.

La quantité de matière sèche totale ingérée est moins élevée quand du laurate est incorporé à la ration. Dans le régime Laurate plus particulièrement, l'aliment concentré expérimental est consommé en proportion plus faible mais de façon non significative. En raison de ces résultats sur la consommation alimentaire, les fourrages non broyés ingérés représentent 20 à $27 \mathrm{p}$. Ioo de la matière sèche totale et l'aliment concentré 73 à $80 \mathrm{p}$. Ioo.

Production et composition du lait (fig. 4).

Bien que les différences sur la production laitière comme dans l'expérience $F$ ne soient pas significatives, il est intéressant de noter l'effet plutôt favorable de 1'acétate et l'effet plutôt dépressif du laurate; ce qui se répercute significativement sur les quantités sécrétées de matières grasses et de matières azotées.

L'acétate et le stéarate élèvent le taux butyreux respectivement de ro et 6 p. Ioo. Dans cette expérience, c'est donc l'acétate et non le stéarate qui est le plus efficace pour augmenter la teneur en matières grasses. Leurs effets semblent être cumulatifs. D'ailleurs, il n'apparaît pas d'interaction significative entre eux. Le laurate a plutôt tendance à diminuer le taux butyreux quand il est ajouté au régime Témoin et surtout lorsqu'il est introduit dans le régime Acétate. 
TABLEAU 3

Consommation alimentaire

(en $\mathrm{kg}$ de matière sèche par jour et par chèvre)

(Expérience $\mathrm{F}$ )

\begin{tabular}{|c|c|c|c|c|c|c|}
\hline & \multicolumn{6}{|c|}{ Régimes } \\
\hline & $\mathbf{T}$ & $\mathbf{A}$ & S & $\mathbf{P}$ & AS & PS \\
\hline $\begin{array}{l}\text { Aliment concentré expéri- } \\
\text { mental } \ldots \ldots \ldots \ldots \ldots \ldots\end{array}$ & 0,92 & 0,91 & 0,90 & 0,85 & 0,92 & 0,90 \\
\hline Ration totale .......... & 2,46 & 2,38 & 2,40 & 2,36 & 2,46 & 2,36 \\
\hline
\end{tabular}

(Expérience C)

\begin{tabular}{|c|c|c|c|c|c|c|}
\hline & \multicolumn{6}{|c|}{ Régimes } \\
\hline & $\mathbf{T}$ & A & $\mathbf{S}$ & $\mathrm{L}$ & AS & AL \\
\hline $\begin{array}{l}\text { Aliment concentré expéri- } \\
\text { mental } \ldots \ldots \ldots \ldots \ldots \ldots \\
\text { Ration totale } \ldots \ldots \ldots \ldots \ldots\end{array}$ & $\begin{array}{l}0,88 \\
1,67\end{array}$ & $\begin{array}{l}0,88 \\
1,73\end{array}$ & $\begin{array}{l}0,87 \\
1,73\end{array}$ & $\begin{array}{l}0,78 \\
1,58\end{array}$ & $\begin{array}{l}0,93 \\
1,77\end{array}$ & $\begin{array}{l}0,86 \\
1,64\end{array}$ \\
\hline
\end{tabular}

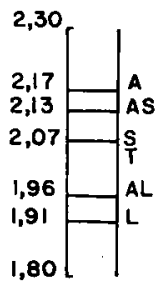

Production laitiēre (kg)

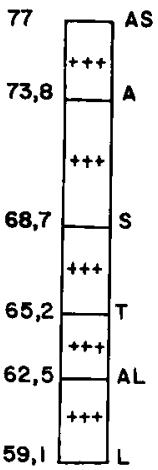

Motières grosses secrótées (g)
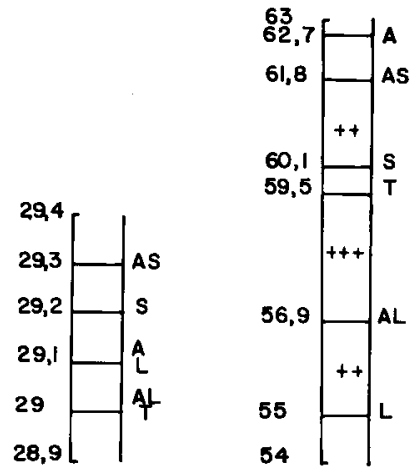

Toux butyreux(g.p 1000$)$

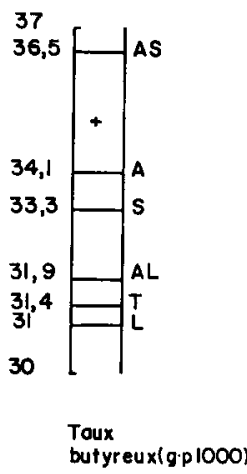

FIG. 4. - Infuence du régime sur la production et la composition du lait

$$
\text { Régimes }\left\{\begin{aligned}
& T \text { : Témoin } \\
& \text { A }: \text { Acétate } \\
& \text { L : Stéarate } \\
& \text { AS : Laurate } \\
& \text { AL : Laurate-Stéarate }
\end{aligned}\right.
$$

Les différents régimes sont classés pour chaque variable selon l'ordre décroissant des résultats.

Lorsque les deux régimes sont séparés :

$$
\operatorname{par}\left\{\begin{array}{r} 
\pm \text { la différence de leurs résultats est significative à } P<0, I \\
+ \text { la différence de leurs résultats est significative à } P<0,05 \\
++ \text { la différence de leurs résultats est significative à } P<0, \text { oI } \\
+++ \text { la différence de leurs résultats est significative à } P<0, \text { oor }
\end{array}\right.
$$

L : signifie à la limite de signification. 
Comme la nature des régimes influence la production laitière et le taux butyreux le plus souvent dans le même sens, leurs effets sur la quantité de matières grasses sécrétées sont encore plus nets. En particulier le stéarate et l'acétate ajoutés à un régime contenant ou non un autre acide augmentent significativement la sécrétion journalière de matières grasses, alors que le laurate l'abaisse toujours de façon significative.

La teneur en matières azotées du lait n'est pas influencée significativement par la nature des régimes.

Pourcentage et quantités d'acides gras sécrétées (fig. 5 et 6).

Dans l'analyse de variance, les effets du régime sur les pourcentages et les quantités sécrétées des acides gras du lait sont tous significatifs, à l'exception de

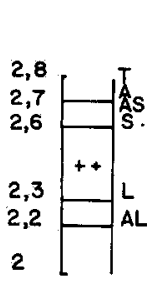

8:0

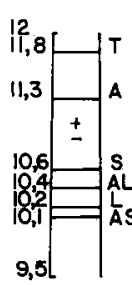

10:0

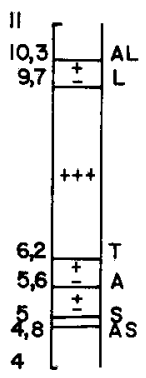

12:0

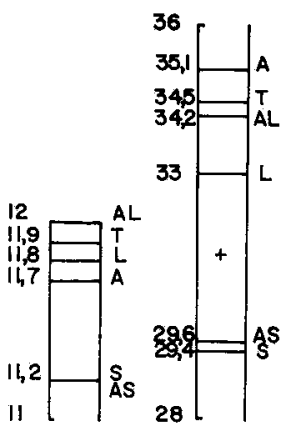

14:0

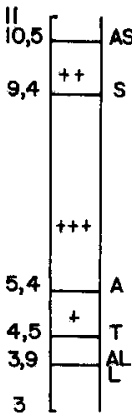

$18: 0$

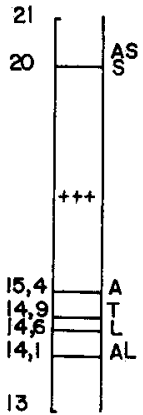

18:1

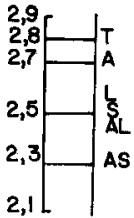

$18: 2$

FIG. 5. - Influence du régime sur la composition en acides gras des lipides du lait

(Expérience C)

(Légende, cf. fig. 4)

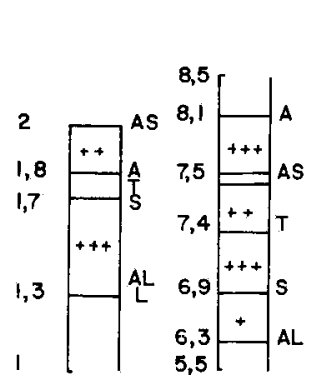

$8: 0$

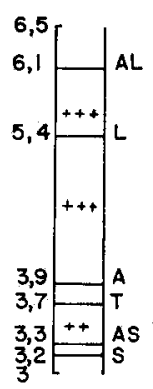

$12: 0$

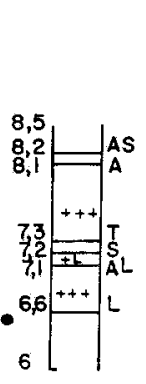

$14: 0$

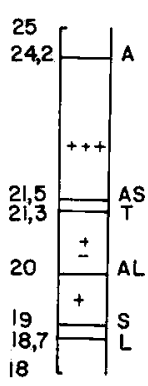

$16: 0$

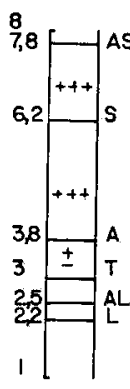

18:0

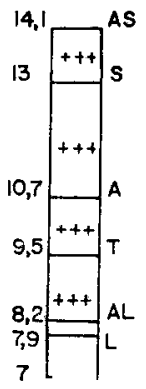

18.1

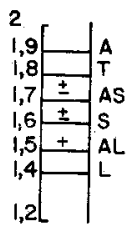

$18 \cdot 2$

FIG. 6. - Influence du régime sur les quantités sécrêtées d'acides gras dans le lait (Expérience $\mathrm{C}$ )

(Légende, cf. fig. 4)

celtui sur le pourcentage de l'acide linoléique. Comme dans l'expérience F, la présence de stéarate dans le régime augmente les pourcentages et les quantités sécrétées d'acides stéarique et oléique. Elle diminue les pourcentages des acides à 
plus courte chaîne, notamment de l'acide palmitique. Mais la quantité sécrétée de ces acides n'est abaissée qu'avec le régime Stéarate. L'apport d'acétate dans le régime Stéarate permet d'améliorer la production d'acides gras à moins de I8 atomes de carbone qui devient légèrement supérieure à celle du régime Témoin.

\section{TABI,EAU 4}

Interactions entre les différents acides ajoutés

\begin{tabular}{|c|c|c|c|c|}
\hline & \multicolumn{2}{|c|}{ Expérience $\mathbf{F}$} & \multicolumn{2}{|c|}{ Expérience C } \\
\hline & $\begin{array}{l}\text { Interaction } \\
\text { Propionate- } \\
\text { Stéarate }\end{array}$ & $\begin{array}{c}\text { Interaction } \\
\text { Acétate- } \\
\text { Stéarate }\end{array}$ & $\begin{array}{c}\text { Interaction } \\
\text { Acetate- } \\
\text { Laurate }\end{array}$ & $\begin{array}{c}\text { Interaction } \\
\text { Acétate- } \\
\text { Stéarate }\end{array}$ \\
\hline \multicolumn{5}{|c|}{ Production et composition du lait } \\
\hline $\begin{array}{l}\text { Production latière } \ldots \ldots \ldots \ldots \\
\text { Taux butyreux } \ldots \ldots \ldots \ldots \\
\text { Matières grasses sécrétées } \ldots \\
\text { Taux matières azotées....... } \\
\text { Matières azotées sécrétées... }\end{array}$ & $\begin{array}{l}\text { NS } \\
\text { NS } \\
\text { NS } \\
\text { NS } \\
\text { NS }\end{array}$ & $\begin{array}{l}\text { NS } \\
\text { NS } \\
\text { NS } \\
\text { NS } \\
\text { NS }\end{array}$ & $\begin{array}{l}\text { NS } \\
\text { NS } \\
\text { NS } \\
\text { NS } \\
\text { NS }\end{array}$ & $\begin{array}{l}\text { NS } \\
\text { NS } \\
\text { NS } \\
\text { NS } \\
\text { NS }\end{array}$ \\
\hline \multicolumn{5}{|c|}{ Pourcentage en acide gras des matières grasses du lait } \\
\hline $\begin{array}{r}8: 0 \\
10: 0 \\
12: 0 \\
14: 0 \\
16: 0 \\
18: 0 \\
18: 1 \\
18: 2\end{array}$ & $\begin{array}{l}\text { NS } \\
\text { NS } \\
\text { NS } \\
\text { NS } \\
\text { NS } \\
\text { NS } \\
\text { NS } \\
\text { NS }\end{array}$ & $\begin{array}{c}\text { NS } \\
\text { NS } \\
\text { NS } \\
\text { NS } \\
+ \\
++ \\
++ \\
\text { NS }\end{array}$ & $\begin{array}{r}\text { NS } \\
\text { NS } \\
+ \\
\text { NS } \\
\text { NS } \\
+ \text { L } \\
\text { NS } \\
\text { NS }\end{array}$ & $\begin{array}{l}\text { NS } \\
\text { NS } \\
\text { NS } \\
\text { NS } \\
\text { NS } \\
\text { NS } \\
\text { NS } \\
\text { NS }\end{array}$ \\
\hline \multicolumn{5}{|c|}{ Quantités d'acides gras sêcrétées dans le lait } \\
\hline $\begin{array}{r}8: 0 \\
10: 0 \\
12: 0 \\
14: 0 \\
16: 0 \\
18: 0 \\
18: 1 \\
18: 2\end{array}$ & $\begin{array}{l}\text { NS } \\
\text { NS } \\
\text { NS } \\
\text { NS } \\
\text { NS } \\
\text { NS } \\
\text { NS } \\
\text { NS }\end{array}$ & $\begin{array}{c}\text { NS } \\
\text { NS } \\
\text { NS } \\
\text { NS } \\
+ \text { L } \\
+ \\
+ \\
\text { NS }\end{array}$ & $\begin{array}{l}\text { NS } \\
\text { NS } \\
\text { NS } \\
\text { NS } \\
\text { NS } \\
\text { NS } \\
\text { NS } \\
\text { NS }\end{array}$ & $\begin{array}{l}\text { NS } \\
\text { NS } \\
\text { NS } \\
\text { NS } \\
\text { NS } \\
\text { NS } \\
\text { NS } \\
\text { NS }\end{array}$ \\
\hline
\end{tabular}

NS : Non significatif.

$+:(P<0,05)$.

$++:(P<0,01)$.

$+++:(P<0,001)$.

L : Limite de signification.

L'acétate seul a tendance à diminuer les proportions des acides caprique, laurique et myristique et à élever ceux des acides palmitique, stéarique et oléique. Mais il augmente les quantités sécrétées de tous les acides quelle que soit la longueur de leur chaîne. De même, ajouté au régime Stéarate son influence sur 
la composition en acides gras est négligeable sauf sur le pourcentage d'acide stéarique, mais sa présence améliore de façon non négligeable la production de tous les acides gras. Des résultats très comparables sont obtenus lorsque 1'acétate est introduit dans le régime Laurate.

L'addition de laurate augmente très nettement le pourcentage et la quantité sécrétée d'acide laurique, mais abaisse de façon le plus souvent significative les pourcentages et les sécrétions journalières de presque tous les autres acides. Seul le pourcentage d'acide myristique ne semble pas être modifié.

Les interactions entre stéarate et acétate et entre laurate et acétate se sont révélées en général non significatives, à l'exception de celles entre laurate et acétate concernant les teneurs en acides laurique et stéarique (tabl. 4). En effet, l'acétate en présence de laurate dans le régime accroît significativement le pourcentage d'acide laurique alors que seul il n'a pas cet effet.

\section{DISCUSSION ET CONCLUSION}

Les deux expériences $\mathrm{F}$ et $\mathrm{C}$ se sont déroulées dans des conditions identiques, au cours de deux années successives; les mêmes chèvres en majorité y participent. Dans ces conditions, les résultats de ces expériences peuvent être comparés entre etux.

Certains effets de l'addition distincte ou cumulée d'acétate et de stéarate au régime Témoin sont comparables dans les deux expériences $\mathrm{F}$ et $\mathrm{C}$. Mais les différences notables enregistrées sur d'autres effets peuvent, le plus souvent, s'expliquer par la nature distincte des régimes Témoin. En effet, d'une part, si les deux régimes Témoin permettent aux chèvres d'ingérer des quantités assez faibles et voisines d'extrait éthéré, celui de l'expérience $C$ est légèrement plus riche en matières grasses en raison de sa forme plus concentrée. D'autre part, dans l'expérience C, la faible proportion d'aliments cellulosiques dans le régime Témoin, le rapport élevé Aliments concentrés/Fourrages grossiers et la présence de pulpes de betteraves déshydratées granulées à la place de fourrages qui tendent à diminuer la proportion d'acide acétique dans le rumen (BATH et Rook, I965), sont autant de facteurs qui contribuent probablement à abaisser l'apport d'acide acétique à la mamelle. De ce fait, il n'est pas étonnant que les pourcentages et les quantités sécrétées des acides en $C_{18}$ soient plus élevés avec le régime Témoin de l'expérience $C$ et que la teneur et la sécrétion de l'ensemble des acides à plus courte chaîne soient inférieures à ceux de l'expérience $\mathrm{F}$.

Un mois avant chaque expérience, les animaux sont habitués au régime Témoin. Lors de la période préexpérimentale de l'expérience $\mathrm{C}$ où les proportions de l'aliment concentré et du foin exclusivement sont modifiées, la production laitière des chèvres baisse sensiblement, alors que le taux butyreux augmente. Cette observation s'est toujours vérifiée chez la chèvre lorsque la proportion de fourrages dans le régime est réduite à 20-25 p. Ioo. Ainsi, cet animal réagit à ce type de régime différemment de la vache qui voit, dans les mêmes conditions, sa production laitière généralement améliorée et le taux butyreux chuter de façon importante (ARMstrong, I968). Il est probable que les phénomènes digestifs et métaboliques 
observés chez la Vache qui reçoit une alimentation riche en aliments concentrés ne se produisent pas dans les mêmes conditions chez la Chèvre. En effet, outre le taux butyreux qui ne diminue pas, le rapport des pourcentages d'acide oléique et d'acide stéarique n'augmente pas comme chez la Vache (JoRGENSEN, I964; STORRY et RoOK, I965; OPSTVEDT et RoNNING, I967). Au contraire, ce rapport passe de 5,05 dans l'expérience $\mathrm{F}$ à 3,3I dans l'expérience $\mathrm{C}$. Ce phénomène montre bien les différences qui peuvent exister entre les réponses des chèvres et des vaches à certains types de régime.

\section{Stéarate}

Dans les deux expériences, le stéarate accroît la production journalière de matières grasses et dans des proportions moindres le taux butyreux. Les pourcentages et les sécrétions des acides stéarique et oléique s'élèvent très nettement, tandis que les teneurs en acides à plus courte chaîne et notamment la teneur en acide palmitique ont tendance à baisser. Ces résultats confirment ceux de DELAGE et FEHR (I967 b) sur chèvres, de STEELE et MOORE (I968), de STEELE (Ig69) et de NoBLE et coll. (I969) sur vaches, qui ajoutent aussi du stéarate à un régime pauvre en lipides. L'acide myristique et l'acide caprylique semblent être moins sensibles à l'effet du stéarate comme cela apparaît déjà dans les résultats de STEELE et MOORE (I968). La teneur en acide linoléique à 1'opposé de celles des autres acides en $\mathrm{C}_{18}$ baisse à la suite d'un apport supplémentaire de stéarate quel que soit le régime Témoin, comme dans les expériences de STEELE et MOORE (I968). D'après DELAGE et FEHR (I967 b), le stéarate alimentaire a tendance à provoquer une diminution du pourcentage des acides essentiels dans les triglycérides du plasma sanguin et ainsi une quantité moindre de ces acides serait prélevée par la mamelle qui ne peut les synthétiser. En revanche, une partie de l'acide oléique du lait provient de l'acide stéarique que la mamelle est capable de desaturer (LAURYSSENS et coll., Ig6I).

L'effet du stéarate est nettement moins marqué dans l'expérience $\mathrm{C}$ que dans l'expérience F. L'ingestion quotidienne de stéarate, inférieure seulement de $8 \mathrm{p}$. roo dans l'expérience $C$, ne peut expliquer totalement sa sécrétion plus faible dans le lait. Les pourcentages des acides en $\mathrm{C}_{18}$ avec le régime Témoin de l'expérience $\mathrm{C}$ sont plus élevés qu'avec celui de l'expérience $\mathrm{F}$. L'apport supplémentaire de stéarate permet aux pourcentages des acides stéarique et oléique d'atteindre des valeurs identiques avec le régime Stéarate de chaque expérience. Dans ces conditions, l'utilisation plus faible dans l'expérience $\mathrm{C}$ du stéarate alimentaire par le tissu sécrétoire de la glande mammaire serait due à un déficit moins accusé en cet acide du régime Témoin. Quelle que soit son importance, 1'augmentation de la sécrétion lipidique de la mamelle s'explique par l'accroissement des quantités sécrétées des acides stéarique et oléique. Mais, elle est toujours liée à des sécrétions sensiblement plus faibles d'acide palmitique et dans une moindre mesure, d'acides à chaîne plus courte. Il est peu probable que l'apport de stéarate représentant dans les deux expériences moins de $3 \mathrm{p}$. Ioo de la matière sèche ingérée et au maximum 6 p. Ioo de l'aliment expérimental, ait une influence sur les fermentations du rumen et qu'en particulier il abaisse la production d'acide acétique. En effet, d'après HAGEMEISTER et KAUFMANN (I969), l'ingestion de $500 \mathrm{~g}$ de stéarate par 
des vaches ne modifie pas la composition en acides gras volatils du rumen. Par ailleurs, ro p. roo de stéarate dans l'aliment concentré, soit une quantité supérieure à celle que nous avons utilisée, n'influence pas dans la panse le pourcentage d'acide acétique et augmente légèrement la teneur en acide propionique au détriment de l'acide butyrique (STEELE et MOORE, I968). Il est plus plausible d'estimer que des quantités importantes d'acides gras à longue chaîne prélevées par la mamelle ralentissent la synthèse intramammaire des acides gras comme le pensent NOBLE et coll. (I969). En effet, BoRTz et coll. (I963) ont montré, sur coupes de foie de rat, que les acides gras à longue chaîne inhibent l'activité de 1'acétyl-CoA carboxylase. Les travaux d'HIBITT et coll. (Ig66) sur homogénats de glande mammaire bovine et ceux de FEHR et coll. (r967), obtenus par infusion intraveineuse sur chèvres, confirmeraient cette interprétation. En réalité, cette synthèse n'est affectée nettement que dans l'expérience F. En effet, les quantités sécrétées de l'ensemble des acides à moins de 18 atomes de carbone avec les régimes Témoin et Stéarate sont plus voisines dans l'expérience $C$ que dans l'expérience $F$. I1 semble bien que le régime Témoin pauvre en fourrages de l'expérience $\mathrm{C}$ puisse avoir aussi une action inhibitrice sur la synthèse des acides gras. En effet, d'après OPSTVEDT et coll. (I967), 1' " acyl-synthétase » du tissu mammaire de la vache avec un régime à base d'aliments concentrés a une activité plus faible que celle de la vache dont le régime est riche en fourrages non broyés.

\section{Laurate}

Dans l'expérience C le laurate a tendance à diminuer le plus souvent de façon significative les quantités sécrétées de lait, de matières azotées, de matières grasses et d'acides gras du lait excepté celle d'acide laurique qui augmente nettement. Ces résultats en accord avec ceux de STEELE et MOORE (I968), bien que leurs conditions expérimentales soient assez éloignées des nôtres, sont probablement dus au niveau de consommation plus faible du régime Laurate et de façon moins accusée du régime Acétate-Laurate. STEELE et MOORE (I968) remarquent aussi que la vache accepte plus difficilement un aliment concentré contenant ro p. Ioo de laurate. En réalité, lorsque les productions sont rapportées à la matière sèche ingérée, l'introduction de laurate dans le régime provoque des performances aussi bonnes, sinon meilleures, que celles des autres régimes. D'ailleurs, le beturre de coprah incorporé dans un régime pauvre en lipides, est très bien accepté par les vaches et favorise le taux butyreux et la sécrétion de lait, de matières grasses et des acides gras du lait (STORRy et coll., I967).

En présence de laurate, le pourcentage et la quantité sécrétée d'acide laurique augmentent significativement, mais dans des proportions nettement plus faibles que ceux des acides en $C_{18}$ à la suite d'un apport alimentaire de stéarate. Or, les niveaux d'ingestion de stéarate et de laurate sont très comparables. Ainsi, la mamelle sécréterait un acide gras d'origine alimentaire d'autant plus facilement que sa chaîne carbonée est longue. BARRY et coll. (I963), en effet, constatent que les acides palmitique et stéarique du plasma sanguin sont prélevés plus intensément par la mamelle que les autres. Il semble, par ailleurs, que si un phénomène d'élongation de chaîne se produit à partir de l'acide laurique, il soit de faible intensité. En effet, le pourcentage et la sécrétion d'acide myristique 
diminuent comme les autres acides, mais dans des proportions plus limitées.

Comme tout acide gras alimentaire, l'acide laurique doit être transporté jusqu'à la mamelle par les $\beta$-lipoprotéines dont le pourcentage en acides en $\mathrm{C}_{18}$ diminuerait consécutivement; ce qui expliquerait les teneurs et les sécrétions plus basses d'acides stéarique, oléique et linoléique dans le lait.

Comme l'interaction entre le laurate et 1'acétate n'est pas significative (tab1. 4), leurs effets sur la sécrétion des acides gras, seraient indépendants. En particulier, l'acétate ajouté à un régime contenant déjà du laurate augmente le pourcentage et la quantité sécrétée d'acide laurique dans le lait. Apparemment les mécanismes de la synthèse lipidique ne sont pas saturés en acide laurique puisque la mamelle peut encore sécréter des quantités supérieures. Ainsi son comportement différent vis-à-vis de l'acide laurique et de l'acide stéarique apportés dans l'alimentation, pourrait être dû en partie à leur prélèvement effectué par la glande en quantité différente (BARRY et coll., I963).

\section{Propionate}

Le propionate dans le régime $\mathrm{P}$ de l'expérience $\mathrm{F}$ a pour effet de diminuer de façon non significative la quantité de matières grasses sécrétées et d'augmenter le taux de matières azotées, ce qui est en accord avec les travaux de Rook et BALCH (rg6r), RooK et coll. (I965) et Wirson et coll. (I967). La très faible diminution de consommation pourrait aussi expliquer ces résultats.

Toutefois, à l'encontre des travaux des autres auteurs, le propionate n'abaisse pas le taux butyreux en raison probablement du régime de référence T de l'expérience $\mathrm{F}$. En effet, la pauvreté en lipides du régime Témoin s'accompagne d'une faible valeur du taux butyreux du lait des chèvres qui le consomment (DELAGE et FEHR, I967 $a$ et c). Par ailleurs, le propionate ne semble pas favoriser la production laitière dans l'expérience $\mathrm{F}$. Comme l'effet du propionate sur la production de lait est très variable suivant les auteurs, WILSON et coll. (Ig67) pensent qu'il agit favorablement sur la production laitière lorsque l'apport énergétique de la ration est insuffisant. Ainsi 1'acide propionique influencerait la sécrétion laitière en tant que fournisseur d'énergie et, par ailleurs, aurait un rôle dépressif sur la synthèse lipidique.

La diminution de la quantité sécrétée d'acides gras à moins de r8 atomes de carbone quoique non significative semble responsable de la baisse peu accusée de la sécrétion lipidique de la mamelle à la suite de l'ingestion de propionate. Le caractère néo-glycogénique de l'acide propionique, généralement admis, pourrait amoindrir l'efficacité des éléments cétogéniques comme l'acide acétique et ainsi ralentir la synthèse des acides gras par la mamelle. Mais ce phénomène reste très limité dans l'expérience $F$ tant dans le cas d'une addition de propionate seul qu'en présence simultanée de stéarate et de propionate.

Des résultats assez différents des nôtres ont été obtenus sur vaches dans des expériences d'infusions ruminales où les quantités de propionate apportées sont en général plus élevées. L,es pourcentages de tous les acides gras, sauf de l'acide palmitique, sont abaissés (STORRY et RooK, I965 a) alors que ØRSKov et coll. (I969) observent un accroissement des pourcentages des acides myristique et stéarique sans augmentation de celui de l'acide palmitique, ce qui se rapprocherait plus de nos propres résultats. 


\section{Acétate}

Comme le stéarate, l'acétate augmente la production de matières grasses et, dans une moindre mesure, le taux butyreux et la production laitière dans les deux expériences $\mathrm{F}$ et $\mathrm{C}$, sans influencer la teneur en matières azotées. Cet effet de l'acétate sur la production laitière et la sécrétion lipidique est généralement reconnu (ARmSTrong, Ig68). En réalité, son influence, qu'il soit ajouté au régime Témoin ou au régime Stéarate, est plus marquante dans l'expérience $\mathrm{C}$ que celle du stéarate, alors que c'est le stéarate dans l'expérience $F$ qui a le plus d'effet. Comme les niveaux d'ingestion d'acétate sont voisins dans les deux expériences, il semble bien que la mamelle utilise d'autant mieux un précurseur des matières grasses du lait qu'elle en est carencée. MIL,LER et ALIEN (I955) et SchELNER (Ig68) affirment qu'à partir d'un certain niveau, l'acétate n'a plus d'effet sur la sécrétion lipidique. Étant donné 1'amélioration relativement faible de la quantité sécrétée de matières grasses observées dans l'expérience $F$, le régime Témoin ne doit pas être loin de fournir une quantité suffisante d'acide acétique à la mamelle.

Les compositions en acides gras des matières grasses du lait obtenues avec les régimes Témoin et Acétate sont très comparables, mais leurs quantités sécrétées sont en général supérieures en présence d'acétate dans le régime. Les sécrétions des acides en $C_{18}$ semblent plus favorisées par 1'addition d'acétate que celles des acides plus courts qui, pourtant, sont synthétisés dans la mamelle à partir d'acide acétique. Ce résultat s'oppose aux travaux de LrNNZELL et coll. (1967), d'Ørskov et coll. (r969) qui, en utilisant des perfusions de mamelle ou des infusions intraruminales, constatent une élévation des sécrétions des acides gras courts et un abaissement de celles des acides à I8 atomes de carbone. Néanmoins, GERSON et coll. (I966), en pratiquant une infusion sur une longue période, observent qu'une faible quantité des acides en $\mathrm{C}_{18}$ du plasma sanguin peut être synthétisée à partir d'acétate, probablement dans des organes comme le foie. Nous pouvons supposer qu'une telle synthèse n'est plus négligeable au cours d'une longue période où le ruminant reçoit un régime carencé en acides gras à I8 atomes de carbone.

L'interaction des effets acétate et stéarate sur les pourcentages et les quantités sécrétées des acides palmitique, stéarique et oléique semble dépendre de la nature du régime Témoin de chaque expérience. Leurs effets sont additifs quand le régime est plus concentré (expérience $\mathrm{C}$ ). La mamelle serait alors sans doute carencée en acide acétique et aussi en acides en $\mathrm{C}_{\mathbf{1 8}}$, mais à un moindre degré comme nous l'avons précisé. En revanche, avec le régime riche en fourrages (expérience F), l'acétate paraît limiter l'influence du stéarate sur les pourcentages et les sécrétions journalières des acides stéarique et oléique, tandis qu'incorporé seul dans le régime, il a un effet favorable, mais non significatif. Dans ce cas, la mamelle serait probablement déficitaire en acides gras en $\mathrm{C}_{18}$ et très peu en acide acétique, comme le laisse supposer le faible effet de l'acétate sur la sécrétion d'acides gras plus courts et 1'accroissement important de la production des acides à 18 atomes de carbone à la suite de l'addition de stéarate. L'effet dépressif du stéarate sur le taux d'acide palmitique dans les matières grasses et sur sa quantité sécrétée par jour est atténué si de 1'acétate est incorporé simultanément à la ration. Ainsi l'inhibition partielle de la synthèse des acides gras dans la mamelle due au stéarate serait en partie levée par l'apport supplémentaire d'acétate. De ce fait, 
la sécrétion des acides en $\mathrm{C}_{18}$ pourrait alors être réduite parce que les possibilités maximales de synthèse lipidique de la mamelle seraient atteintes, comme semblent l'indiquer les valeurs du taux butyreux.

Ainsi, il apparaît assez clairement, à la vue des résultats de ces deux expériences, que la mamelle de chèvre utilise d'autant mieux 1'acétate et le stéarate ajoutés aux rations pour synthétiser les matières grasses du lait, que le régime lui en fournit de faibles quantités. Dans ces conditions, 1'utilisation par la glande mammaire de l'un de ces précurseurs des lipides du lait dépendrait de la nature du régime. Lorsque le régime carence nettement la mamelle à la fois en acides gras longs et en acide acétique, les effets de leur addition simultanée dans la ration s'ajouteraient l'un à l'autre; ils agiraient donc de façon indépendante. Par contre, si le régime est peu déficitaire en l'un de ces précurseurs, leurs effets cumulés pourraient être atténués, donc n'être pas complètement additifs. Dans ce cas, l'interaction de leurs effets serait apparente. Comme les acides gras à chaîne longue limiteraient la synthèse des acides gras notamment de l'acide palmitique à partir d'acide acétique, il semble que ces acides gras longs auraient plus de chances d'avoir un effet plus prononcé que l'acide acétique sur la sécrétion lipidique de la mamelle de ruminant. Cependant, ces mécanismes sont susceptibles d'être perturbés par le double effet de l'acide acétique et aussi de l'acide propionique sur le métabolisme de la mamelle. En effet, une concurrence pourrait s'établir entre leur rôle spécifique de fournisseur d'énergie et leur rôle spécifique dans la synthèse lipidique. Ainsi, le niveau énergétique de la ration interviendrait de façon non négligeable dans l'utilisation des précurseurs des lipides du lait par la mamelle de ruminant.

Rę̧u pour publication en novembre 1971.

SUMMARY

\section{ISOLATED AND COMBINED EFFECTS OF ACETIC, PROPIONIC, LAURIC AND STEARIC ACIDS ON LIPID SECRETION OF THE GOAT UDDER}

Two experiments, $\mathrm{F}$ and $\mathrm{C}$, were carried out in latin-square design on I 8 goats. The isolated and combined effects of acetic, propionic, lauric, and stearic acids have been studied on milk yield and composition, the fatty acid composition of milk fat, and the daily secretions of these fatty acids.

Six diets were given to the goats in each experiment. The control diet $\mathrm{T}$ in experiment $\mathrm{F}$ is low in fat and high in forage ; that of experiment $C$ is low in forage and a little higher in fat. In both experiments, sodium acetate, ethyl stearate, acetate and stearate are added to diet $T$, and in experiment $F$ diet $T$ is supplemented with sodium propionate, propionate and stearate, while in experiment $C$, it is supplemented with ethyl laurate, acetate and laurate.

Adding stearate to diet $\mathrm{T}$ increases fat percentage, butterfat yield, and the percentage and quantity of stearic and oleic acids secreted. The amounts of short-chain acids decrease. Dietary stearate seems to slow down intra-udder fatty-acid synthesis. These effects are less mar ked in experiment $\mathrm{C}$ than in experiment $\mathrm{F}$.

Laurate tends to diminish milk, fat, and protein yield as well as the amounts of fatty acids secreted, with the exception of lauric acid which significantly increases. The longer is the chain of a dietary fatty acid, the easier is it secreted by the udder. The effects of laurate and acetate seem independent.

Propionate has little effect. Due to a lower secretion of fatty acids containing less than I 8 carbon atoms, it slightly reduces the quantity of fats secreted. In other respects, it increases the percentage of protein. 
Acetate, like stearate, increases the butterfat content and fat yield more significantly in experiment $C$ than in experiment $F$. The udder seems to better utilize a fat precursor in which it is deficient. After acetate is added, the quantity of all the fatty acids secreted in milk increases, particularly those of $\mathrm{C}_{18}$. The interaction of acetate and stearate seems to depend on the type of control diet. When the diet deprives the udder both of acetic acid and long fatty acids, the effects of acetate and sterate are significant. If the diet is not deficient in acetic acid, the cumu. lative effects diminish and are no longer significant. As long-chain fatty acids could limit intraudder synthesis when acetic is involved, these long dietary fatty acids would have a more pronounced effect on lipid secretion in the goat udder than acetic acid.

\section{RÉFÉRENCES BIBLIOGRAPHIQUES}

ARmstrong D. G., 1968. The amount and physical form of feed and milk secretion in cows. Proc. Nutr. Soc., 27, 57-65.

Barry J. M., Bartley W., Linzell J. L., Robinson D. S., 1963. The uptake from the blood of triglyceride fatty acids of chilomicra and low density lipoproteins by the mammary gland of the goat. Biochem. J., 89, 6-II.

BAth I. H., Rook J. A. F., 1965. The evaluation of cattle foods and diets in terms of the ruminal concentration of volatile fatty acids. II. Roughage and succulents. J. Agric. Sci., 64, 67-75.

Bortz W., Agraham S., Chaikoff J. L., r963. Localization of the block in lipogenesis resulting from feeding fat. J. Biol. Chem., 288, 1266-1272.

Cochran W. G., Cox G. M., r957. Experimental designs. $2^{e}$ ed., John Wiley and Sons, N. Y.

Craig B. M., Murty N. L., I959. Quantitative fatty acid analysis of vegetable oils by gas-liquid chromatography. J. Amer. Oil. Chem. Soc., 16, 549-554.

Delage J., Fehr P. M., rg67 a. Influence des lipides alimentaires sur la sécrétion des acides gras par la mamelle de chèvre. I. Influence de la teneur du régime en lipides sur le taux butyreux du lait et sa composition en acides gras. Ann. Biol. anim. Biochim. Biophys., 7, 437-444.

Delage J., Fehr P. M., 1967 $b$. Influence des lipides alimentaires sur la sécrétion des acides gras par la mamelle de chèvre. II. Influence de l'addition d'acides gras en $\mathrm{C}_{1 \mathrm{~g}}$ à des régimes pauvres en lipides, sur la composition lipidique du plasma sanguin et du lait. Ann. Biol. Anim. Biochim. Biophys., 7, 445-457.

Delage J., Fehr P. M., x967 c. Influence de la composition lipidique du régime sur le taux butyreux du lait de chèvre. C. R. Acad. Sci., 264, $981-984$, Série D.

Fehr P. M., Delage J., Le Bars H., r967. (Résultats non publiés).

Fehr P. M., Disset R., 1969. Peut-on alimenter rationnellement les chèvres? Rev. Élevage, $45^{\mathrm{e}} \mathrm{n}^{\circ}$ spéc. "Moutons et Chèvres ", r45-15I.

Fehr P. M., Delage J., 197o. Effets distincts et cumulés des acides acétique et stéarique sur la sécrétion des acides gras par la mamelle de chèvre. C. R. Acad. Sci., 271, 237-240 (Série D).

Folley S. J., MacNaught M. L., I96r. Biosynthesis of milk fat, Milk. The mammary gland and its secretion, vol. I, 44I-482, éd. by Kon S. K. and Cowie A. T. Academic Press inc. New York.

Garton G. A., 1963. The composition and biosynthesis of milk lipids. J. Lipid Res., 4, $237-254$.

Gerson T., Wilson G. F., Singh H., Shorland F. B., 1966. Origin of the glyceride fatty acids of milk fat. J, Dairy Sci., 49, 680-68r.

Hagemeister H., Kaufmann W., I969. Das Fettsaüremuster des Milchfettes bei Zufütterung von Stearinsaüre. Milchwissenschaft, 24, 654-657.

Hrbits K. G., 1966. Some factors involved in the control of fatty acid synthesis in the lactating bovine mammary gland. Biochim. Biophys. Acta, 116, 56-66.

JonEs E. A., 1969. Reviews of the progress of dairy science. Section A. Recent developments in the biochemistry of the mammary gland. J. Dairy Res., 36, 145-167.

Jorgensen N. A., 1964. Ration effects on rumen acids, blood components and milk composition in dairy cows. Diss. Abstr., 25, $2128-2129$.

Lauryssens M., Verbecke R., Peeters G., I96r. Metabolism of stearate ${ }^{14} \mathrm{C}$ in the isolated cow'udder. J. Lipid Res., 2, 383-388.

Linzell J. L., Annison E. F., Farakerley S., Leng R. A., 1967. The incorporation of acetate, stearate and $\beta$-hydroxybutyrate into milk fat by the isolated perfused mammary gland of the goat. Biochem. J., 104, 34-42.

Miller W. J., Allen N. N., I955. The effect of sodium acetate feeding on milk and fat yield, blood sugar and blood ketones of dairy cows. J. Dairy Sci., 38, 310-312.

Noble R. C., Steele W., Moore J. H., I969. The effects of dietary palmitic and stearic acids on milk fat composition in the cow. J. Dairy Res., 36, 375-38o. 
Opstvedt J., Ronning M., 1967. Effect upon lipid metabolism of feeding alfalfa hay or concentrate ad libitum as the sole feed for milking cows. J. Dairy Sci, 50, 345-354.

Ørskov E. R., Flatt W. P., Moe P. M., Munson A. W., Hemken R. W., Katz I., I969. The influence of ruminal infusion of volatile fatty acids on milk yield and composition on energy utilization by lactating cows. $B r . J . N$ utr., $23,443-453$.

Rook J.A. F., BALCh C. C., I96r. The effects of intraruminal infusions of acetic, propionic and butyric acids on the yeld and composition of the milk of the cow, Brit. J. Nutr., 15, 36r-369.

Rook J. A. F., Balch C. C., Johnson V. W., I965. Further observations on the effects of intraruminal infusions of volatile fatty acids and of lactic acid on the yield and composition of the milk of cow. Brit. J. Nutr., 10, 93-99.

Rose-Gottlieb, I926. Méthodes officielles américaines d'analyse du lait. Le lait, 6, 54-74.

Schelner G., I968. Natriumacetate-Eine Möglichkeit zur steigerung der Milch und Milchfettleistung des Rindes. Tierzucht, $\mathrm{n}^{\circ} 2$, 90-92.

SteEle W., MOORE J. H., I968. The effects of a series of satured fatty acids in the diet on milk fat secretion in the cow. J. Dairy Res., 35, 36I-370.

SteEle W., I969. The effects of dietary palmitic and stearic acids on milk yield and composition in the cow. J, Dairy Res., 36, 369-373.

Storry J. E., Rook J. A. F., I965. Effect in the cow of intraruminal infusions of volatile acids and of lactic acid on the secretion of the component fatty acids of the milk fat and on the composition of blood. Biochem. J., 96, 210-2I7.

Storry J. E., Rook J. A. F., I965. The effects of a diet low in hay and hight in flaked maize on milk fat secretion and on the concentrations of certain constituents in the blood plasma of the cow. Brit. J. Nutr., 19, IoI-109.

Storry J. E., Rook J. A. F., Hall A. J., I967. The effect of the amount and type of dietary fat on milk fat secretion in the cow. Brit. J. Nutr., 21, 425-438.

Wilson G. F,, Davey A. W. F., Dolby R. M., I967. Milk composition as affected by intraruminal infusion of volatile fatty acids to cows on a restricted ration. N.Z. J. A gric. Res., 10, 215-225. 